\title{
On the Equality of Rank of a Fifth-Idempotent Matrix
}

\author{
Lin Deng", Feng Zhang, Jinli Xu \\ Department of mathematics, Northeast Forestry University, Harbin, China, 150040
}

*Corresponding Author: Lin Deng, Department of mathematics, Northeast Forestry University, Harbin, China, 150040

Abstract: The equality of rank a fifth-idempotent matrix is established by means of elementary transformation and properties of idempotent matrix.

Keywords: fifth-idempotent matrix, rank, equality.

\section{INTRODUCTION}

Proposition $1 A^{5}=A \Leftrightarrow \operatorname{rank}(A)+\operatorname{rank}\left(E-A^{4}\right)=n$

Proof: Since the elementary transformation of a matrix does not change the rank of the matrix, the following equality can be obtained.

$$
\operatorname{rank}\left[\begin{array}{cc}
A & \\
& E-A^{4}
\end{array}\right]=\operatorname{rank}\left[\begin{array}{cc}
A & \\
A^{4} & E-A^{4}
\end{array}\right]=\operatorname{rank}\left[\begin{array}{cc}
A & A \\
A^{4} & E
\end{array}\right]=\operatorname{rank}\left[\begin{array}{cc}
A-A^{5} & 0 \\
A^{4} & E
\end{array}\right]=\operatorname{rank}\left[\begin{array}{cc}
A-A^{5} & 0 \\
0 & E
\end{array}\right]
$$

Therefore $A^{5}=A \Leftrightarrow \operatorname{rank}(A)+\operatorname{rank}\left(E-A^{4}\right)=n$

Proposition $2 A^{5}=A \Rightarrow \operatorname{rank}\left(A^{a}\right)+\operatorname{rank}\left(E-A^{4}\right)^{b}=n, \forall a, b \in N^{+}$

Proof: On the one hand, by $A^{5}=A$, we have $A\left(E-A^{4}\right)=0$, So for every positive integer, we have $A^{a}\left(E-A^{4}\right)=0$. With the help of the property of matrix multiplication operation, we can get $\operatorname{rank}\left(A^{a}\right)+\operatorname{rank}\left(E-A^{4}\right) \leq n$.

On the other hand, The minimum polynomial of matrix $A$ obtained from $A^{5}=A$ is the factor of polynomial $\lambda^{5}-\lambda$. Therefore, the minimum polynomial of $\boldsymbol{A}$ has no multiple roots, so $A$ can be diagonalized.

For every positive integer $a, b$, there exists an invertible matrix $P$ such that the following equation holds.

$$
P\left[A^{a}+\left(E-A^{3}\right)^{b}\right] P^{-1}=P A^{a} P^{-1}+P\left(E-A^{3}\right)^{b} P^{-1}=\left(P A P^{-1}\right)^{a}+\left[E-\left(P A P^{-1}\right)^{3}\right]^{b}
$$

It is not hard to get $\operatorname{rank}\left[A^{a}+\left(E-A^{4}\right)^{b}\right]=n$. 
Hence it follows that $n=\operatorname{rank}\left[A^{a}+\left(E-A^{4}\right)^{b}\right] \leq \operatorname{rank}\left(A^{a}\right)+\operatorname{rank}\left(E-A^{4}\right)^{b} \leq n$

Therefore $\operatorname{rank}\left(A^{a}\right)+\operatorname{rank}\left(E-A^{4}\right)^{b}=n$

Proposition $3 A^{5}=A \Rightarrow \operatorname{rank}(A)+\operatorname{rank}\left(E-A^{4}+A^{3}\right)=n+\operatorname{rank}\left(A^{4}\right)$

Proof The following equation can be obtained from elementary transformation.

$$
\begin{aligned}
& \operatorname{rank}\left[\begin{array}{cc}
A & \\
& E-A^{4}+A^{3}
\end{array}\right]=\operatorname{rank}\left[\begin{array}{ll}
A & \\
A & E-A^{4}+A^{3}
\end{array}\right]=\operatorname{rank}\left[\begin{array}{cc}
A & A^{4}-A^{3} \\
A & E
\end{array}\right] \\
& =\operatorname{rank}\left[\begin{array}{cc}
A-A^{5}+A^{4} & A^{4}-A^{3} \\
0 & E
\end{array}\right]=\operatorname{rank}\left[\begin{array}{cc}
A-A^{5}+A^{4} & 0 \\
0 & E
\end{array}\right]
\end{aligned}
$$

Substituting $A^{5}=A, \operatorname{rank}\left[\begin{array}{cc}A-A^{5}+A^{4} & 0 \\ 0 & E\end{array}\right]=\operatorname{rank}\left[\begin{array}{cc}A^{4} & 0 \\ 0 & E\end{array}\right]$,

Therefore $\operatorname{rank}\left[\begin{array}{cc}A & \\ & E-A^{4}+A^{3}\end{array}\right]=\operatorname{rank}\left[\begin{array}{cc}A^{4} & 0 \\ 0 & E\end{array}\right]$.

That is to say $\operatorname{rank}(A)+\operatorname{rank}\left(E-A^{4}+A^{3}\right)=n+\operatorname{rank}\left(A^{4}\right)$

Conversely, it does not necessarily hold true. For example, $A=(1+\sqrt{3}) E$ $\operatorname{rank}(A)+\operatorname{rank}\left(E-A^{4}+A^{3}\right)=2 n=n+\operatorname{rank}\left(A^{4}\right), \quad$ but obviousiy there are $A^{5} \neq A$.

Proposition $4 A^{5}=A \Rightarrow \operatorname{rank}\left(E-A^{4}+A^{3}\right)=\operatorname{rank}\left(A^{4}\right)+\operatorname{rank}\left(E-A^{4}\right)$

Since $A^{5}=A \quad, \quad$ from proposition 1 and 2, $\operatorname{rank}(A)+\operatorname{rank}\left(E-A^{4}\right)=n \quad$, $\operatorname{rank}(A)+\operatorname{rank}\left(E-A^{4}+A^{3}\right)=n+\operatorname{rank}\left(A^{4}\right)$.

So we can get $\operatorname{rank}\left(E-A^{4}+A^{3}\right)=\operatorname{rank}\left(A^{4}\right)+\operatorname{rank}\left(E-A^{4}\right)$.

But the same, based on $\operatorname{rank}\left(E-A^{4}+A^{3}\right)=\operatorname{rank}\left(A^{4}\right)+\operatorname{rank}\left(E-A^{4}\right)$, we can not get $A^{5}=A$. For example,

$$
A=\left[\begin{array}{lll}
1 & 1 & 0 \\
0 & 0 & 1 \\
0 & 0 & 0
\end{array}\right] A^{3}=A^{4}=\left[\begin{array}{lll}
1 & 1 & 1 \\
0 & 0 & 0 \\
0 & 0 & 0
\end{array}\right]
$$

$\operatorname{rank}\left(E-A^{4}+A^{3}\right)=3=\operatorname{rank}\left(A^{4}\right)+\operatorname{rank}\left(E-A^{4}\right)$

But $A^{5}=\left[\begin{array}{lll}1 & 1 & 1 \\ 0 & 0 & 0 \\ 0 & 0 & 0\end{array}\right] \neq\left[\begin{array}{lll}1 & 1 & 0 \\ 0 & 0 & 1 \\ 0 & 0 & 0\end{array}\right]=A$

According to the definition of the fifth-idempotent matrix and its operation, the following properties of the fifth-idempotent matrix can be given. 


\section{Proposition 4}

(1)If the fifth-idempotent matrices $A, B$ are commutative, then $A B$ is also a fifth-idempotent matrix.

(2) If $A$ is a fifth-idempotent matrix, then $A^{4}$ is an idempotent matrix.

(3) If $A$ is a fifth-idempotent matrix, $E-A^{4}$ is an idempotent matrix.

(4) If $A$ is a fifth-idempotent matrix, then for any positive integer, there are

$$
A^{n}=\left\{\begin{array}{c}
A, 4 \mid n-1 \\
A^{2}, 4 \mid n-2 \\
A^{3}, 4 \mid n-3 \\
A, 4 \mid n
\end{array}\right.
$$

Proposition 5 If $A, B$ are all fifth-idempotent matrices, the following equality is satisfied

(1) $\operatorname{rank}\left(A^{4}+B^{4}\right)=\operatorname{rank}\left[\begin{array}{cc}A^{4} & B^{4} \\ B^{4} & 0\end{array}\right]-\operatorname{rank} B^{4}=\operatorname{rank}\left[\begin{array}{cc}B^{4} & A^{4} \\ A^{4} & 0\end{array}\right]-\operatorname{rank} A^{4}$

(2) $\operatorname{rank}\left(A^{4}+B^{4}\right)=\operatorname{rank}\left[A^{4}-A^{4} B^{4}, B^{4}\right]=\operatorname{rank}\left[B^{4}-B^{4} A^{4}, A^{4}\right]$

(3) $\operatorname{rank}\left(A^{4}+B^{4}\right)=\operatorname{rank}\left(A^{4}-A^{4} B^{4}-B^{4} A^{4}+B^{4} A^{4} B^{4}\right)+\operatorname{rank} B^{4}$

(4) $\operatorname{rank}\left(A^{4}+B^{4}\right)=\operatorname{rank}\left(A^{4}-A^{4} B^{4}-B^{4} A^{4}+A^{4} B^{4} A^{4}\right)+\operatorname{rank} A^{4}$

(5) $\operatorname{rank}\left(A^{4}+B^{4}\right)=\operatorname{rank}\left[\begin{array}{ccc}A^{4} & B^{4} & 0 \\ B^{4} & 0 & A^{4}\end{array}\right]=\operatorname{rank}\left[\begin{array}{ll}A^{4} & B^{4}\end{array}\right]$

(6)If $a_{1}, a_{2}$ are two non-zero real numbers and $a_{1}+a_{2} \neq 0$, then

$\operatorname{rank}\left(a_{1} A^{4}+a_{2} B^{4}\right)=\operatorname{rank}\left(A^{4}+B^{4}\right)$.

Theorem $1 A \in P^{n \times n}, f(x), g(x) \in P[x]$ is a polynomial with any number greater than 1 . Let $d(x)=(f(x), g(x)), m(x)=[f(x), g(x)]$, then

$\operatorname{rankf}(A)+\operatorname{rankg}(A)=\operatorname{rankd}(A)+\operatorname{rankm}(A)$.

Corollary $A \in P^{n \times n}, f(x) \in P[x]$ is a polynomial with any number greater than 1 . Let $d(x)=\left(f(x), x-x^{5}\right)$ and $m(x)=\left[f(x), x-x^{5}\right]$, then

$\operatorname{rankf}(A)+\operatorname{rank}\left(A-A^{5}\right)=\operatorname{rankd}(A)+\operatorname{rankm}(A)$. 
With the help of corollary we can get if $\boldsymbol{A}$ is a fifth-idempotent matrix, then $\operatorname{rankf}(A)=\operatorname{rankd}(A)+\operatorname{rankm}(A)$.

This corollary shows that there are also many rank eigenvalues of ffifth-idempotent matrices.

\section{Theorem 2}

$A \in P^{n \times n}, t \geq 1 \in N^{+}, \operatorname{rank}(A)+\operatorname{rank}\left(A^{t}-A^{t+4}\right)=\operatorname{rank}\left(A^{t}\right)+\operatorname{rank}\left(A-A^{5}\right)$.

Proof: When $\mathrm{t}=1$, the equation clearly holds.

Let $t>1, f(x)=x^{t}, \quad g(x)=x-x^{5}$, By simple calculation we get $(f(x), g(x))=x$ $[f(x), g(x)]=x^{t}-x^{t+4}$. By the above we can get the following equation.

$\operatorname{rank}(A)+\operatorname{rank}\left(A^{t}-A^{t+4}\right)=\operatorname{rank}\left(A^{t}\right)+\operatorname{rank}\left(A-A^{5}\right)$

\section{REFERENCES}

[1] Idempotent Matrix[M]// The Concise Encyclopedia of Statistics. 2008.

[2] Baksalary O M, Bernstein D S, Trenkler G. On the equality between rank and trace of an idempotent matrix[J]. Applied Mathematics \& Computation, 2010, 217(8):4076-4080.

[3] BAKSALARY, Jerzy K, BAKSALARY, et al. Idempotency of linear combinations of an idempotent matrix and a tripotent matrix[J]. Linear Algebra \& Its Applications, 2002, 354(1):21-34.

[4] Hartwig R E, Trenkler G. Properties of Idempotent Matrix[J]. Econometric Theory, 1997, 13(4):606-606.

Citation: Lin Deng, et.al., (2019). On the Equality of Rank of a Fifth-Idempotent Matrix. International Journal of Scientific and Innovative Mathematical Research (IJSIMR), 7(7), pp. 8-11. http://dx.doi.org/ 10.20431/2347-3142.0707003

Copyright: () 2019 Authors, this is an open-access article distributed under the terms of the Creative Commons Attribution License, which permits unrestricted use, distribution, and reproduction in any medium, provided the original author and source are credited. 